\title{
Détermination du GHB dans les poils pubiens par chromatographie gazeuse cou- plée à la spectrométrie de masse en tandem
}

\section{Determination of $G H B$ in pubic hair by $G C-M S / M S$}

\section{Capucine DUHEM $^{(1)}$, Marion VILLAIN ${ }^{(2)}$, Anne Laure PELISSIER ${ }^{(3)}$, Vincent CIRIMELE ${ }^{(2)}$, Pascal KINTZ ${ }^{(1,2) *}$}

(1) Institut de Médecine Légale, Strasbourg (2) Laboratoire ChemTox, Illkirch Graffenstaden

(3) Institut de Médecine Légale, Marseille

* Auteur à qui adresser la correspondance : Pascal KINTZ, Laboratoire ChemTox, 3, rue Grüningen 67400 Illkirch Graffenstaden - Tél : +33 (0)3904033 32 - Fax : +33(0)390400541

E-mail : pascal.kintz@wanadoo.fr

(Reçu le 19 août 2004 ; accepté après modifications le 12 octobre 2004)

\section{$R E ́ S U M E$}

L'acide gamma hydroxybutyrique ou GHB a été dosé dans les poils pubiens d'un homme décédé d'un surdosage. C'est le premier cas mortel rapporté en France. Après avoir été décontaminés et segmentés, les poils pubiens ont été incubés avec $10 \mathrm{ng}$ de $G H B-d_{6}$ (utilisé comme étalon interne) dans de la soude à $0,01 N$. Après neutralisation, acidification et extraction dans l'acétate d'éthyle, les composés sont silylés par un mélange contenant du (N-(ter-butyldimethylsilyl)-Nmethyltrifluoroacetamide ou MTBSTFA. Le GHB (m/z 275 donnant 143, 147 et 149) et le GHB-d $(\mathrm{m} / 2281$ donnant 147, 148 et 149) ont été identifiés et quantifiés par CPGSM/SM. Les concentrations trouvées dans les différents segments de poils pubiens sont comprises entre 19,4 et 25,0 $\mathrm{ng} / \mathrm{mg}$ alors que les concentrations physiologiques sont inférieures à $2 \mathrm{ng} / \mathrm{mg}(n=14)$.

\section{MOTS CLÉS}

$G H B$, poils pubiens, surdosage.

\section{SUMMARY}

$G H B$ has been determined in pubic hair from a man deceased from a GHB overdose. This is the first French fatal case that is reported in the literature. Pubic hair was collected during autopsy, decontaminated, segmented and incubated in $0.01 \mathrm{~N}$ sodium hydroxyde in presence of $10 \mathrm{ng}$ of $G H B \cdot d_{6}$. After neutralisation, acidification and extraction in ethyl acetate, the target compounds are derivatizated using MTBSTFA. GHB $(\mathrm{m} / \mathrm{z} 275$ to $143,147,149)$ and $G H B-d_{6}$ $(\mathrm{m} / \mathrm{z}, 281$ to $147,148,149)$ were identified and quantified by GC-MS/MS.

While the physiological concentrations are lower than 2 $n g / m g(n=14)$, the concentrations in the three pubic hair segments were $19.4,22.6$ and $25.0 \mathrm{ng} / \mathrm{mg}$, respectively.

GHB, pubic hair, overdose. 


\section{Introduction}

L'acide gamma hydroxybutyrique (GHB) est naturellement présent chez l'homme. Utilisé depuis les années 60 comme anesthésique, le GHB a été ces dernières années détourné de son usage premier. En effet depuis 1980, l'usage de GHB s'est développé dans le milieu du culturisme. Il est utilisé comme substituant aux anabolisants stéroïdiens pour son action stimulante sur l'hormone de croissance, action non démontrée scientifiquement à ce jour.

Puis le GHB a fait son apparition dans les clubs de nuit et raves-parties pour ses propriétés euphorisantes, désinhibitrices et sédatives. Il est alors utilisé à des dosages variant de 30 à $40 \mathrm{mg} / \mathrm{kg}(1,2)$.

Plus récemment, le GHB a été retrouvé dans des affaires de soumission chimique où ses propriétés sédatives et amnésiantes ont été mises à profit.

Depuis une dizaine d'années, le GHB est dosé dans le sang et l'urine en routine. Mais la faible fenêtre de détection du GHB dans ces deux milieux, ainsi que la formation post-mortem de GHB a conduit certains laboratoires à utiliser une autre matrice, les cheveux. Celle-ci présente l'avantage de pouvoir mettre en évidence le GHB plusieurs semaines après les faits. Les cheveux peuvent être utilisés comme un calendrier historique et permettent de détecter une exposition unique au GHB $(3,4)$. Il n'y a pas de publication à ce jour sur la détection du GHB dans les poils pubiens.

Le premier décès français par GHB a permis au laboratoire d'analyser cette molécule dans les poils pubiens en appliquant une méthode d'analyse similaire à celle utilisée pour les cheveux. Pour cela, les concentrations physiologiques du GHB ont d'abord été mesurées chez 14 volontaires.

\section{Présentation du cas médi- co-légal}

Un homme de 43 ans est retrouvé chez lui, par ses proches, inanimé. Malgré l'intervention rapide du service des urgences, il décède. Cet homme était connu des services de police comme étant consommateur de stupéfiants.

A l'autopsie, le légiste note une congestion pulmonaire, et aucune trace de violence n'est mise en évidence. Des prélèvements de sang, urine, liquide gastrique, bile et humeur vitrée sont effectués pour les analyses toxicologiques et conservés à $4^{\circ} \mathrm{C}$. Les cheveux faisant défaut, des poils pubiens sont prélevés et conservés à température ambiante. Du fait d'une orientation parfai- te des poils lors de leur recueil, il a été possible, par la suite, de les segmenter.

\section{Matériels et méthodes}

\section{Standards et réactifs}

La pyridine et l'iodure d'ammonium $\left(\mathrm{NH}_{4} \mathrm{I}\right)$ proviennent de Prolabo (Paris, France). Le MTBSTFA -1\% TBDMCS (N-(ter-butyldimethylsilyl)- $\mathrm{N}$-methyltrifluoroacetamide $+1 \%$ t-butyldimethylchlorosilane) vient de Sigma-Aldrich (Saint Quentin Fallavier, France).

L'agent dérivant utilisé est un mélange MTBSTFA/TBDMCS/ $\mathrm{NH}_{4} \mathrm{I} /$ acétonitrile/pyridine dans les proportions suivantes : 891/9/5/70/30 $(\mathrm{v} / \mathrm{v} / \mathrm{m} / \mathrm{v} / \mathrm{v})$.

Le GHB et GHB- $\mathrm{d}_{6}$ ont été achetés chez Promochem (Molsheim, France).

\section{Extraction du GHB des poils pubiens}

Les poils pubiens $(5 \mathrm{mg})$ décontaminés par le dichlorométhane et segmentés sont placés dans un tube à bouchon vissant, comprenant $0,5 \mathrm{ml}$ de $\mathrm{NaOH}$ à $0,01 \mathrm{~N}$ et $20 \mu \mathrm{l}$ de GHB- $\mathrm{d}_{6}$ à $0,5 \mathrm{mg} / \mathrm{l}$, puis incubés une nuit à $56^{\circ} \mathrm{C}$. Le milieu est ensuite neutralisé par $50 \mu \mathrm{l}$ d' $\mathrm{HCl}$ à $0,1 \mathrm{~N}$, puis acidifié par $50 \mu \mathrm{l}$ d' $\mathrm{H}_{2} \mathrm{SO}_{4}$ à $0,01 \mathrm{M}$. Pour l'extraction, $2 \mathrm{ml} \mathrm{d}$ 'acétate d'éthyle sont ajoutés. Après agitation (15 min à $100 \mathrm{mot} / \mathrm{min}$ ), centrifugation (15 min à $3000 \mathrm{tr} / \mathrm{min}$ ), la phase organique est prélevée puis évaporée. La dérivation se fait avec $25 \mu \mathrm{l}$ d'agent dérivant pendant $75 \min$ à $75^{\circ} \mathrm{C}$.

\section{Instrumentation}

Le système utilisé est constitué d'un chromatographe en phase gazeuse (Hewlett Packard 5890) couplé à un spectromètre de masse en tandem (Finnigan TSQ 700).

$2 \mu \mathrm{l}$ de l'échantillon préparé sont injectés en mode splitless dans une colonne Optima 5MS ( $30 \mathrm{~m}, 0,25$ $\mathrm{mm}$ ID $, 0,25 \mu \mathrm{m}$ d'épaisseur de film). La température de l'injecteur est de $270^{\circ} \mathrm{C}$. Le four suit le cycle de température suivant : température initiale $70^{\circ}$ pendant une minute puis augmentation de la température de $15^{\circ}$ $\mathrm{C} /$ min jusqu'à $295^{\circ} \mathrm{C}$. Le gaz vecteur utilisé est l'hélium, avec une pression en tête de colonne à 10 psi.

Le spectromètre de masse est en mode impact électronique et les acquisitions sont de type SRM. La tension du multiplicateur d'électrons est réglée à 1900 Volts.

Les ions parents $(\mathrm{m} / \mathrm{z}: 275$ et 281$)$ sont sélectionnés dans le premier quadripôle, les ions fils correspondant $(\mathrm{m} / \mathrm{z}: 143,147,149$ et $147,148,149)$ sont sélectionnés dans le troisième quadripôle après collision avec l'argon $(0,65 \mathrm{mT})$. 


\section{Résultats et discussion}

\section{Validation}

Les temps de rétention et les ions du GHB sont rapportés dans le tableau I.

L'ensemble des résultats concernant les paramètres de la validation est donné dans le tableau II .

Tableau I : Temps de rétention et fragments ioniques $(\mathrm{m} / z)$ des différentes molécules recherchées.

\begin{tabular}{|l|l|l|}
\hline & GHB & GHB-d \\
\hline $\operatorname{Tr}(\mathrm{min})$ & 11,43 & 11,39 \\
\hline $\mathrm{m} / \mathrm{z}$ & $\underline{143}, 147,149$ & $147,148,149$ \\
\hline
\end{tabular}

Les ions soulignés ont été utilisés pour la quantification.

Tableau II : Paramètres de validation de la méthode.

\begin{tabular}{|l|l|l|}
\hline Linéarité (de 0,5 à $50 \mathrm{ng} / \mathrm{mg})$ & $\mathrm{y}=0,0274 \times-0,1506 \quad \mathrm{r}^{2}=0,987$ \\
\hline Rendement d'extraction (2 $\mathrm{ng} / \mathrm{mg})$ & $44,9 \%$ \\
\hline \multirow{2}{*}{ Coefficient de variation intra-série } & $\mathrm{C}=0,77 \mathrm{ng} / \mathrm{mg}(\mathrm{n}=8)$ & $15,8 \%$ \\
\cline { 2 - 3 } & $\mathrm{C}=0,51 \mathrm{ng} / \mathrm{mg}(\mathrm{n}=8)$ & $10,0 \%$ \\
\hline
\end{tabular}

$\mathrm{C}=$ concentration moyenne en $\mathrm{GHB}$

Tableau III : Concentrations physiologiques de GHB dans les poils pubiens de 14 volontaires sains des deux sexes.

\begin{tabular}{|c|c|}
\hline Sujet & GHB en ng/mg \\
\hline 1 & 0,74 \\
\hline 2 & 0,36 \\
\hline 3 & 1,05 \\
\hline 4 & 0,30 \\
\hline 5 & 0,31 \\
\hline 6 & 1,55 \\
\hline 7 & 0,58 \\
\hline 8 & 1,21 \\
\hline 9 & 0,56 \\
\hline 10 & 0,76 \\
\hline 11 & 1,10 \\
\hline 12 & 1,10 \\
\hline 13 & 1,13 \\
\hline 14 & 0,38 \\
\hline
\end{tabular}

Tous les poils humains contenant une concentration physiologique de GHB, la linéarité a été faite dans la mélanine $(0,5 \mathrm{mg}$, permettant de mimer la matrice protéique) sur 7 points, de façon à couvrir des concentrations finales comprises entre 0,5 et $50 \mathrm{ng} / \mathrm{mg}$. L'équation de la droite obtenue est : $y=0,0274 x$ 0,1506 avec un coefficient de corrélation $\mathrm{r}^{2}=0,987$.

Les coefficients de variation sont de $15,8 \%$ pour une concentration moyenne de $0,77 \mathrm{ng} / \mathrm{mg}$ et de $10,0 \%$ pour une concentration de $0,51 \mathrm{ng} / \mathrm{mg}$ (répétabilité sur deux pools de 8 échantillons identiques). Le rendement relatif d'extraction est de $44,9 \%$. La limite de détection de la méthode est de $0,1 \mathrm{ng} / \mathrm{mg}$.

Le GHB dérivé est stable au moins 24 heures.

De façon à établir les concentrations physiologiques en GHB, les poils pubiens de 14 témoins ont été analysés. Il s'agissait de 7 femmes et 7 hommes âgés de 24 à 55 ans. La moyenne des concentrations est de $0,8+/-0,4$ $\mathrm{ng} / \mathrm{mg}$, avec une concentration maximale de 1,55 $\mathrm{ng} / \mathrm{mg}$ et une concentration minimale de $0,3 \mathrm{ng} / \mathrm{mg}$ (tableau III), sans qu'il soit possible d'établir une différence de concentration entre les 2 sexes. La sensibilité de la méthode mise en place est amplement suffisante pour la détection du GHB dans les poils pubiens.

\section{Cas médico-légal}

Les poils pubiens analysés ont été coupés en 3 segments $(0$ à $0,8 \mathrm{~cm}, 0,8$ à $1,6 \mathrm{~cm}$ et 1,6 à $2,4 \mathrm{~cm})$. Les concentrations mesurées dans les segments sont de 25,0, 22,6 et 19,4 ng/mg. Ces concentrations sont nettement supérieures aux concentrations physiologiques établies précédemment et démontrant une exposition régulière et répétée au GHB. La figure 1 représente le chromatogramme obtenu après analyse d'un segment de la victime.

L'usage de GHB par la victime a été confirmé par les analyses effectuées dans le sang cardiaque (3385 mg/l) et l'urine ( $33727 \mathrm{mg} / \mathrm{l})$. L'analyse toxicologique complète a mis, par ailleurs, en évidence dans le sang de l'ecstasy (MDMA) à la concentration de $144 \mathrm{ng} / \mathrm{ml}$ (5). Les décès par intoxication au GHB sont rares, peu de cas ont été décrits dans la littérature. 


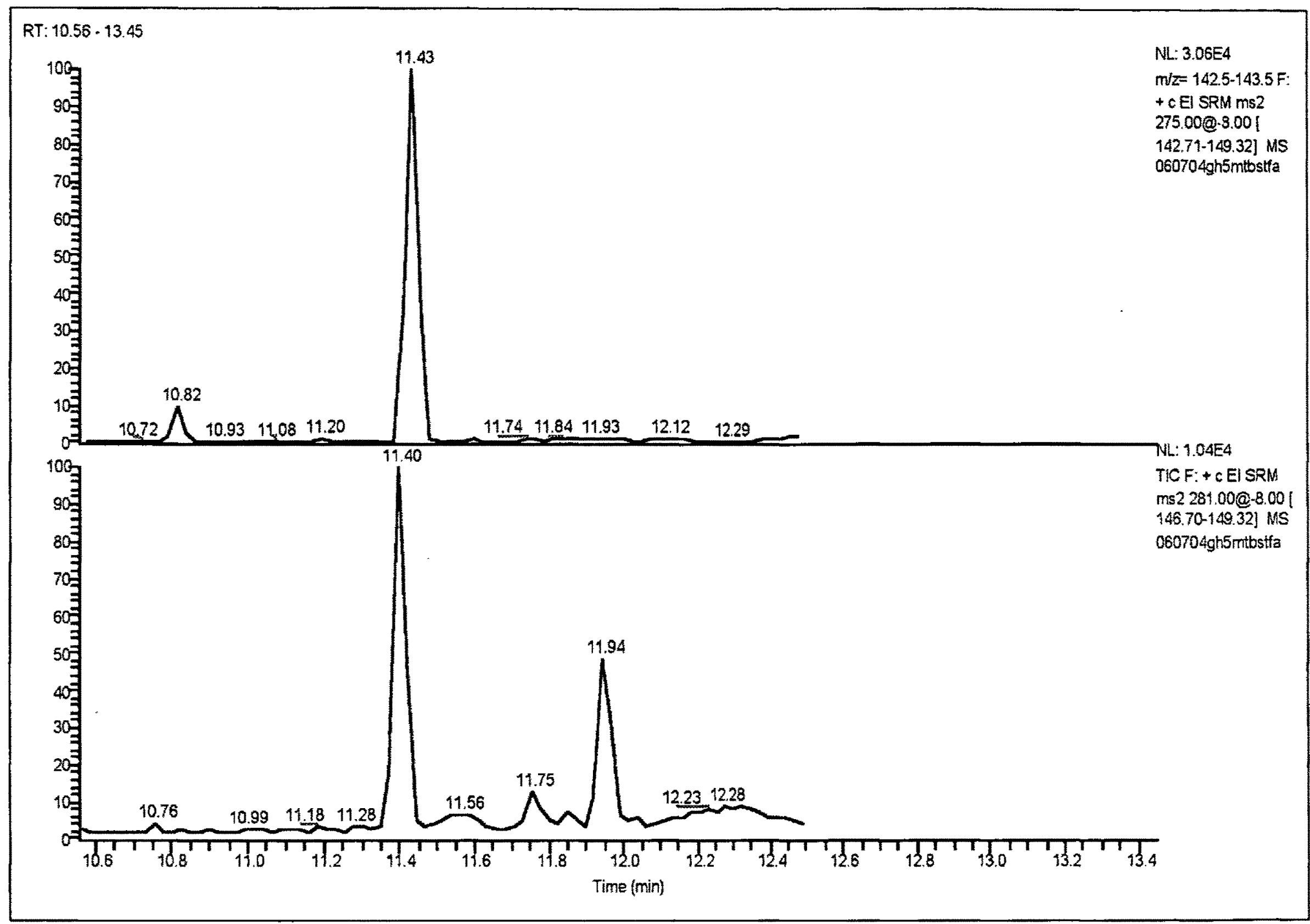

Figure 1 : Chromatogramme obtenu après analyse d'un segment de la victime (GHB dosé à 19,4 ng/mg et élué en 11,43 min). En haut, $G H B$, en bas, $G H B-d_{6}$.

\section{Conclusion}

L'analyse du GHB dans les cheveux a prouvé son intérêt dans deux situations médico-légales. En premier lieu, dans les affaires de soumission chimique, où la segmentation des cheveux permet non seulement d'associer la concentration de GHB de chaque segment à une période définie, mais aussi de montrer l'abstinence à la substance avant et après les faits. Par ailleurs, chez le consommateur chronique, l' analyse des cheveux permet d'obtenir un marqueur biologique pour caractériser un usage régulier.

Les poils pubiens peuvent aussi être utilisés dans ce second cas, notamment à défaut de cheveux. Ils pourraient également confirmer les résultats obtenus dans les cheveux.

\section{Références}

1. Ghysel MH. Le GHB : L'acide Gamma Hydroxy Butyrique. Revue de la littérature. Toxicorama 1999 ; $11: 1-11$.

2. Deveaux M. L'acide gamma hydroxybutyrique (GHB) : un vieux produit, de nouveaux problèmes. Ann. Toxicol. Anal, 2002, 14, 417-23.

3. Kintz P. , Cirimele V., Jamey C., Ludes B. Testing for GHB in hair by GC/MS/MS after a single exposure. Application to document sexual assault. J. Forensic Sci. $2003 ; 48: 195-200$.

4. Goullé JP., Chèze M., Pépin G. Détermination of endogenous levels of GHB in human hair. Are there possibilities for the identification of GHB administration through hair analysis in case of drug-facilitated sexual assault ? J. Anal. Toxicol. $2003 ; 27: 574-80$.

5. Kintz P., Villain M., Pelissier A-L., Jamey C., Duhem C., Sanvoisin A., Leonetti G. Unusually high concentrations in a fatal GHB case. J. Anal. Toxicol, sous presse. 